\title{
Neural Activation during Covert Processing of Positive Emotional Facial Expressions
}

\author{
R. J . Dolan,*,† P. Fletcher,* J . Morris,* N. Kapur,キ J . F. W. Deakin,§ and C. D. Frith*,ף \\ *Wel Icome Department of Cognitive Neurol ogy, I nstitute of Neurology, Queen Square, London WC1N 3BG; †Royal FreeH ospital School of \\ Medicine, Roland Hill Street, London NW3; ¥Wessex Neurological Centre, Southampton General Hospital and Department of Psychol ogy, \\ University of Southampton, Southampton SO16 6YD; §School of Psychiatry and Behavioural Sciences, Rawnsley Building, Manchester \\ Royal Infirmary, Oxford Road, Manchester M 13 9WL; and IDepartment of Psychol ogy, University College London, Gower Street,
} London WC1E 6BT, United Kingdom

Received April 30, 1996

Lesion studies indicate distinct neural systems for recognition of facial identity and emotion. Split-brain experiments also suggest that emotional evaluation of a stimulus can occur without conscious identification. The present study tested a hypothesis of a differential neural response, independent of explicit conscious mediation, to emotional compared to nonemotional faces. The experimental paradigm involved holding in mind an image of a face across a 45-s delay while regional cerebral blood flow was measured using positron emission tomography. Prior to the delay, a single face was presented with an explicit instruction to match it to one of two faces, photographed at different angles from the target face, presented at the end of the delay. Repeated blood flow measures were obtained while subjects held happy or neutral faces in mind or during a neutral control fixation condition without initial face presentation. The representation of emotional faces over a delay period, compared to either the nonemotional or the fixation condition, was associated with significant activation in the left ventral prefrontal cortex, the left anterior cingulate cortex, and the right fusi form gyrus. The findings support our hypothesis of a differential neural response to facial emotion, independent of conscious mediation, in regions implicated in the processing of faces and of emotions. 1996 Academic Press, Inc.

\section{INTRODUCTION}

Signaling emotions using the face is a critical channel of social information processing which endows humans with prodigious abilities in perceiving the dispositions and intentions of others, an ability often referred to as social cognition (Brothers, 1990). The perception of facial expression is an early developmental event with infants displaying a capacity for imitative facial expression at $36 \mathrm{~h}$ and a capacity for facial gesture at 2 weeks (Meltzoff and Moore, 1977; Sagi and Hoffman, 1976). The early, and invariant, emergence in development of both imitative and expressive facial behavior and the circumscribed repertoire of facial emotions across cultures suggests these capacaties are both innate and hardwired (Ekman, 1992).

Cognitive models of face recognition are derived largely from experiments requiring overt responses. However, there is extensive evidence of covert facial recognition from studies in both normals and prosopagnosics (Bruce and Young, 1986; DeHaan et al., 1987; Bruyer et al., 1983; Rizzo et al., 1987; Tranel and Damasio, 1985; Barrett and Rugg, 1989; Barrett et al., 1988; Uhl et al., 1990). Selective lesions may also result in an inability to recognize facial expressions (Shuttleworth et al., 1982). These observations provide an expanded model of face recognition involving dissociable pathways mediating overt recognition, damaged in prosopagnosia, and covert recognition (Bauer, 1986).

Single cell recordings in the primate indicate that face recognition involves circumscribed brain regions in the inferior temporal cortex (Moran and Desimone, 1985; Perrett et al., 1982; Desimone and Gross, 1979). In humans, lesions of the inferior temporal cortex lead to impairments on tests of face recognition (Meadows, 1974) while in healthy subjects functional imaging studies, across a range of experimental paradigms, have identified a neuronal response to faces in the fusiform gyrus (Haxby et al., 1994; Sergent et al., 1994; Courtney et al., 1996). The neural structures involved in processing emotional salience in faces are less well defined although the ventral and orbital prefrontal cortex are seen as critical to emotional regulation (Damasio and Van Hoesen, 1983; Rolls et al., 1994; Damasio et al ., 1990).

Functional imaging studies involving the overt processing of facial emotion have implicated a range of structures including the cingulate cortex, the inferior frontal gyrus, and the right lateral occipital cortex 
(Sergent et al., 1994; George et al., 1993b). The aim in the present study was to identify brain systems involved in the covert processing of facial emotion. In studies of normal subjects involving the induction of both happy and sad moods we have demonstrated activation in the ventral prefrontal cortex suggesting an involvement of this region in the representation of emotional states (Baker et al., 1996). Conversely, an extensive neuropsychological literature implicates the ventral occipital region in the processing of facial identity (Damasio et al., 1982; Meadows, 1974). On this basis we hypothesized activation of the ventral prefrontal cortex in the representation of facial emotion and the medial occipital gyrus in the representation of faces irrespective of emotion.

\section{METHODS}

\section{Subjects}

Eight normal healthy right-handed male subjects (mean age 23, range 20 to 27 years) were included in the study. Handedness was determined according to the Edinburgh I nventory (Oldfield, 1971). These subjects were free of significant past or concurrent illness. All subjects provided written informed consent for the study which was approved by the local hospital ethics committee and ARSAC (UK).

\section{Psychological Task}

To ensure that the processing of emotional faces would be incidental we designed an experimental paradigm that explicitly emphasized a mnemonic requirement. This strategy also minimized any conscious processing such as the linguistic categorization of facial expressions. The task was based upon a delayed matching task in which subjects were instructed to hold in mind, over a 45-s interval, an image of a face in order to match it to a choice of two faces presented at the end of the delay. Each face was presented on a screen $45 \mathrm{~cm}$ in front of the subjects for $2 \mathrm{~s}$ prior to activation of the scan. During the scan, subjects fixated while consciously holding in mind the target face. At the end of scan acquisition two faces appeared on the screen and subjects were required to point to the target face. Both the distractor and the target faces were photographed at different angles from the target face. The target and distractor faces were matched on variables such as sex, age, and race. The position of the target face was systematically varied. Performance on the task was measured as the percentage of target faces identified correctly.

At the end of the scanning session subjects were debriefed with reference to their spontaneous observations on the study. All subjects reported doing the task by holding a representation of the face in mind over the delay period. Subjects also reported that they found this task difficult and that they tended tolose the image of the face toward the second half of the delay period. None of the subjects were consciously aware that the study embodied a component that involved emotion or emotional expression. The subjects did not report any factor that may have helped their performance across conditions such as facial type. Subjects were subse quently asked to classify the target faces into emotional types and all subjects were reliably able to classify the faces as neutral or happy.

Stimuli were presented in a counterbalanced order. F our of thescans involved the presentation of faces that embodied expressions of happiness while another four scans involved faces that were expressively neutral. In the control condition no faces were presented prior to the scan and subjects fixated at a target point in the center of the screen alone. For the control condition subjects were required at the end of the scanning period to point to the older of two faces that appeared on the screen. The accuracy of delayed facial matching was recorded for the active conditions.

\section{Data Analysis}

Scans were obtained using a CTI Model 953B PET Scanner (CTI, Knoxville, TN) with collimating septa retracted. Vol unteers received a 20-s intravenous bolus of $\mathrm{H}_{2}{ }^{15} \mathrm{O}$ at a concentration of $55 \mathrm{MBq} \mathrm{ml}$ and a flow rate of $10 \mathrm{ml} / \mathrm{min}$ through a forearm cannula. The data were analyzed with statistical parametric mapping (SPM 95 software from the Wellcome Department of Cognitive Neurol ogy, London, UK) implemented in Matlab (Mathworks, Inc., Sherborn, MA). Statistical parametric mapping combines the general linear model (to create the statistical parametric map or SPM) and the theory of Gaussian fields to make statistical inferences about regional effects (F riston et al., 1991, 1994).

The scans from each subject were realigned using the first as a reference. Following realignment all images were spatially normalized, using nonl inear transformation, into a standard space and smoothed (Friston et al., 1995a). As a final preprocessing step, the images were smoothed using an isotropic Gaussian kernel.

The condition, subject, and covariate effects (global blood flow) were estimated according to the general linear model at each voxel (Friston et al., 1995b). Totest hypotheses about regionally specific condition effects, the estimates were compared using linear compounds or contrasts. The resulting set of voxel values for each contrast constitutes a statistical parametric map of the t statistic, SPM (t). The SPM (t ) were transformed to the unit normal distribution [SPM(z) and thresholded at 3.09 (or $\mathrm{P}=0.001$ uncorrected for multiple comparisons)]. 


\section{TABLE 1}

Coordinates and Significance of Activations for the Comparison of the Combined Faces with the Control Condition (1a) and the E motional with the Nonemotional Condition (1b)

\begin{tabular}{|c|c|c|c|c|}
\hline & $x$ & $y$ & $z$ & $Z$ value \\
\hline \multicolumn{5}{|c|}{ (a) F aces versus control } \\
\hline Left inferior frontal gyrus & -44 & 22 & -8 & 3.38 \\
\hline Right inferior frontal gyrus & 28 & 28 & -12 & 3.23 \\
\hline Left anterior cingulate gyrus & -12 & 26 & 28 & 3.21 \\
\hline Right fusiform gyrus & 44 & -44 & -12 & 3.08 \\
\hline \multicolumn{5}{|c|}{ (b) Emotional versus nonemotional } \\
\hline Left inferior frontal gyrus & -50 & 26 & 0 & 3.50 \\
\hline Left anterior cingulate gyrus & -12 & 26 & 28 & 3.53 \\
\hline Right fusiform gyrus & 44 & -42 & -12 & 3.26 \\
\hline Thalamus & 8 & -10 & 12 & 3.19 \\
\hline
\end{tabular}

\section{RESULTS}

\section{Performance}

For all delayed matching conditions performance was at ceiling with no errors recorded for either the emotional or the nonemotional conditions.

\section{Functional Imaging Data}

\section{All Faces (Emotional and Nonemotional) versus the Control Condition}

The comparison of the combined faces condition with the reference condition was associated with a number of regional activations which survived a preset threshold of significance $(P<0.001)$. The coordinates of the maxima for these foci are detailed in Table $1 a$ and were centered on the left inferior frontal gyrus (BA 47), the left anterior cingulate cortex (BA 32), and the right fusiform gyrus (BA 20).

\section{Emotional Faces versus Nonemotional Faces and Control Condition}

A comparison of the emotional with the nonemotional faces conditions was associated with a profile of activation similar to that of the combined faces versus control condition. Significant activations were again observed in the left inferior frontal gyrus (BA 47), the left anterior cingulate cortex (BA 32), and the right fusiform gyrus (BA 20). The coordinates of maximal activation for this comparison are shown in Table $1 \mathrm{~b}$ and the areas of significant difference are displayed as SPMs in Fig. 1. A comparison of the emotional faces with the fixation control condition was associated with maximal activation in the left inferior prefrontal cortex $(P<0.0005)$, the right inferior prefrontal cortex ( $P<0.001)$, the right fusiform gyrus $(P<0.001)$, and the left anterior cingulate cortex $(P<0.001)$.

\section{Nonemotional Faces versus the Control Condition}

A comparison of the nonemotional faces with the control condition was associated with a single focus of activation $(P<0.001)$ in the left inferior parietal lobule (BA 40). The mean regional cerebral blood flow (rCBF) response at the pixel coordinates of maximal intensity in the left inferior prefrontal cortex, the anterior cingulate cortex, and the right fusiform cortex for each condition is presented in Fig. 2.

\section{DISCUSSION}

The human face is a crucial component of social communication particularly in the ability to infer the intentions of others. Psychol ogical models of face recognition postulate dissociable processes related to identity and expression (Bruce and Young, 1986). It has been suggested that "the face stimulus is immediately and obligatorily transformed into the representation of a person (with dispositions and intentions) before having access to consciousness" (Brothers, 1990). The present study suggests that the neural response to emotion in faces is both covert and obligatory. There was no requirement for conscious processing yet there were clear differences in neural activation when representing happy compared to neutral faces. The virtually identical pattern of neuronal response in the combined faces condition compared to that of the emotional faces condition alone implies that emotional salience is an important determinant of the magnitude of the associated neuronal response.

The restriction to one class of emotional expression in the present experiment was determined by two considerations. First, the restriction was a constraint of the delayed matching task methodology. The study design provided a means whereby faces could be presented to subjects without a requirement for them to be explicitly aware of the experimental question. To obtain reasonable statistical power in a task involving the maintenance of a single facial representation over the entire scanning period it was necessary to have multiple repeats of each condition. Second, the choice of happy facial expressions was dictated by empirical evidence of a processing advantage over other forms of facial expression (Kirita and Endo, 1995; Ekman et al., 1982; Kirouac and Dore, 1983). In terms of signal characteris-

FIG. 1. SPM of foci of maximum significance for the comparison of emotional and nonemotional conditions. The foci are shown superimposed on a magnetic resonance structural scan that itself has been transformed to standard stereotactic space. The regions highlighted are (a) the left inferior frontal gyrus, the left anterior cingulate cortex, and (b) the right fusiform gyrus. 
a
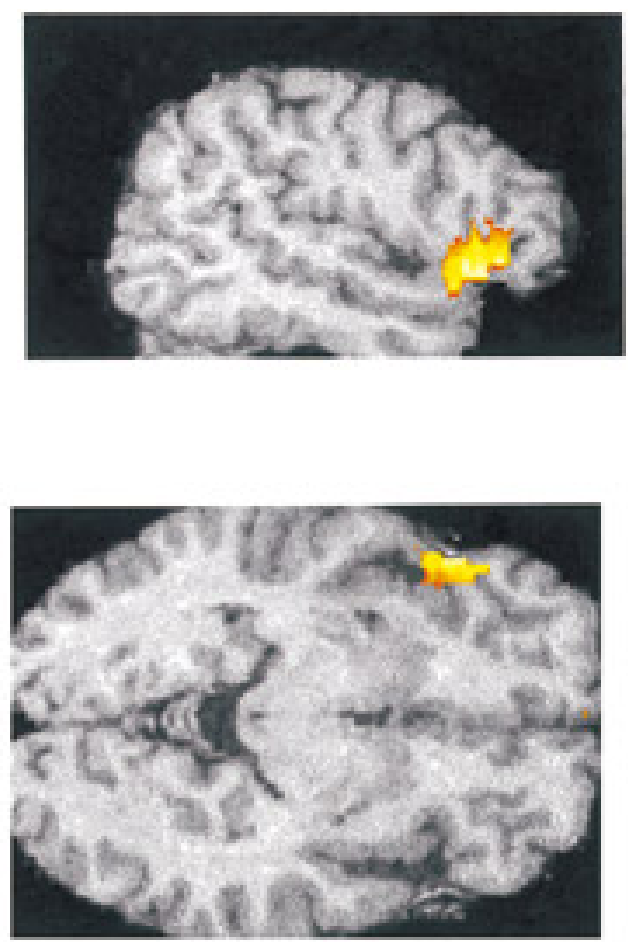

b
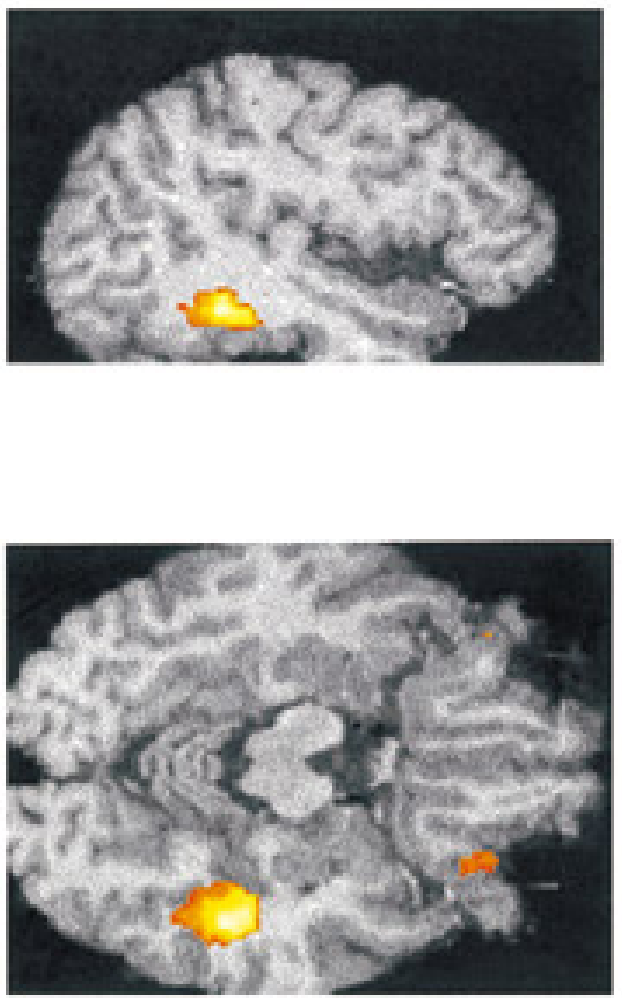
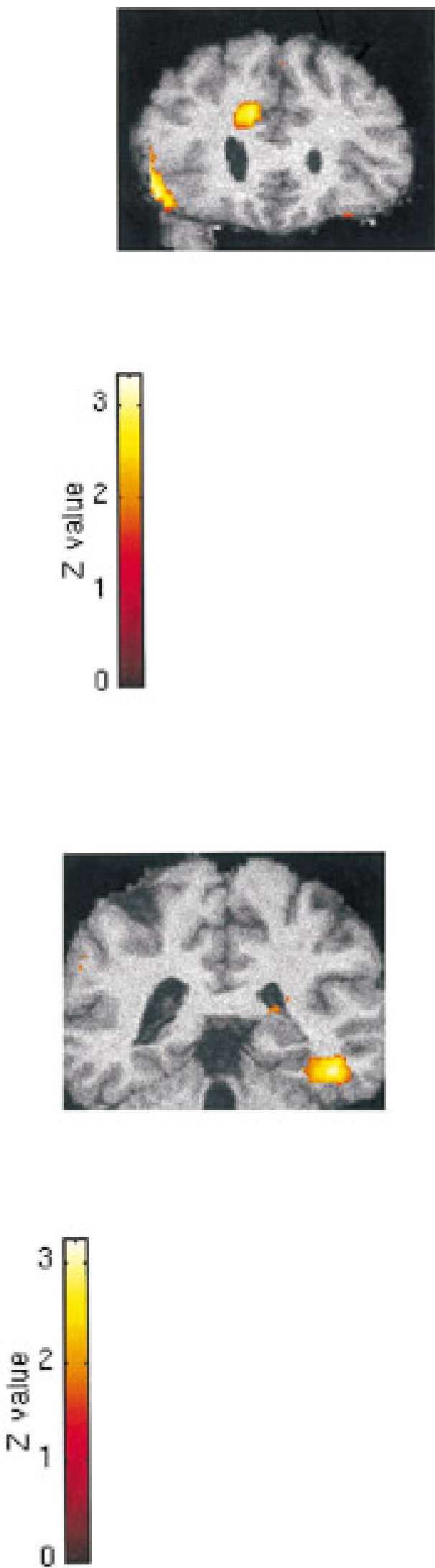

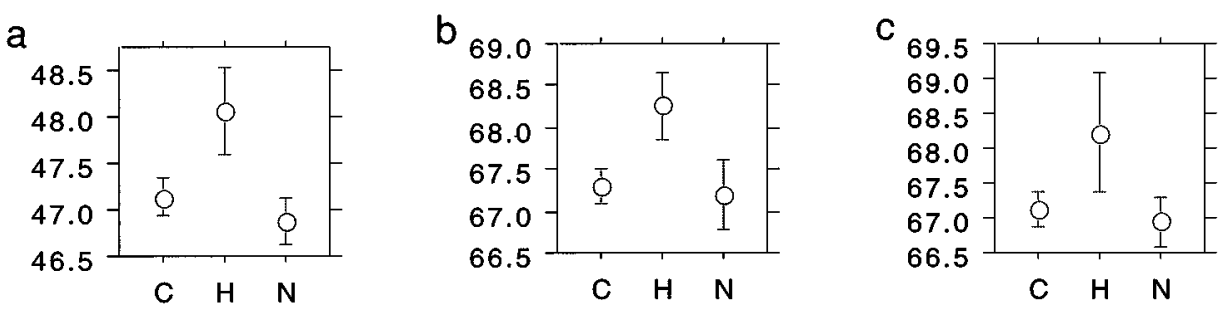

FIG. 2. The mean rCBF responses, at the coordinates of maximal significance in the emotional versus nonemotional condition, are displayed as plots (mean and standard error of the mean) for each condition for (a) the left inferior prefrontal cortex, (b) the left anterior cingulate, and (c) the right fusiform.

tics the smile is the simplest emotional expression but one of the most complex in terms of signal value (Ekman and Friesen, 1982).

The regional activation in the right fusiform gyrus, seen for the combined and emotional faces comparison with the control condition, was not unexpected. An extensive literature implicates the ventral occi pitotemporal cortex in the processing of faces in both human and nonhuman primate. Prosopagnosia, a disorder of facial recognition, is seen following bilateral lesions to the ventral temporal cortex (Damasio et al., 1982; Meadows, 1974). Single-cell recordings in a ventral occipital region in the nonhuman primate have identified units specialized for coding faces or features of faces (Perrett et al., 1992, 1982). In humans direct neurophysiological measures, based upon recordings from extracellular electrodes, have described a negative surface potential (N200) evoked by faces, but not other classes of stimuli, centered on the fusiform and inferior temporal gyri while electrical stimulation of the same region producing a temporary inability to name a familiar face (Allison et al., 1994).

Functional imaging data have provided direct in vivo evidence for involvement of the ventral temporal cortex in face processing. In a face matching task significant activations in the right fusiform gyrus, virtually identical to the regions activated in the present study, have been reported (Haxby et al., 1994). Similarly, in a face working-memory task, activations in the right fusiform and the inferior frontal cortex and the anterior cingulate gyrus have been observed (Courtney et al., 1996). Whether neutral faces alone were used in the latter study is unclear. In a study requiring the explicit processing of facial identity and emotion, activations were noted in the right fusiform gyrus in the identity condition and in the cingulate in the emotion condition but much more caudal to the focus identified in the present study (Sergent et al., 1994). Our finding of unilateral activation in the right fusiform gyrus, however, is consistent with evidence that impaired facial recognition may also be seen after unilateral right temporal lobe lesions (De Renzi, 1996; Landis et al., 1986).

The evidence from the present study of right fusiform activation in the representation of emotional faces is consistent with evidence of a relative superiority of the right hemisphere in processing facial effect that is independent of its superiority in processing facial identity (Bowers and Heilman, 1985). This suggests an involvement of this region in processes additional to that of identity. Alternatively, the happy expression may be more attentionally engaging. The coactivation of the cingulate cortex would support this notion and also explain the absence of fusiform or cingulate activations in the comparison of nonemotional faces with the control condition. The focus identified in the present study may thus represent a region involved in a number of processes that includes identity and expression. A suggested role in processing the salience of facial representations is strengthened by observations that unilateral lesions of the right temporal cortex, insufficient to impair recognition of identity, may impair recognition of facial expression (Bowers and Heilman, 1984; Rapsak et al., 1993). Whether particular forms of expressive salience are processed is an open question particularly in light of findings regarding selective impairment in fear recognition following bilateral amygdala lesions (Adol phs et al., 1995).

The cingulate cortex was strongly activated in the comparison of emotional and nonemotional faces. In humans the anterior cingulate cortex has heterogenous functions (Devinsky and Luciano, 1993). Its activation in the emotional faces condition may relate to the possibility that emotional faces automatically engage attention. A specific role for the cingulate in emotion is an alternative, though complementary, explanation. Lesions of the cingulate, as well as producing a state of akinetic mutism, lead to states of emotional indifference (Damasio and Van Hoesen, 1983). A specific role in emotion is suggested by findings of a decrease in function in this area during states of clinical depression (Bench et al., 1992). Its role in phonation in primates and degrees of facial expressiveness in humans suggests a specificity in relation to expressive aspects of emotion (Muller-Preuss and J urgens, 1976; Von Cramon and J urgens, 1983).

The activation of the ventral prefrontal cortex was a specific prediction of the study. Electrophysiological evidence in the monkey has provided evidence that neurons in the inferior frontal cortex show selective 
responses to faces (Wilson et al., 1993). I ts involvement in emotional processing in humans is supported by observations that lesions involving this region are associated with a failure of the expected augmentation of skin conductance responses to visual stimuli with a high emotional valence (Damasio et al., 1991). The activation of the inferior frontal convexity provides converging evidence of a specific role in emotional processing. Lesions in this region impair the production of emotional, but not nonemotional, facial expressions (Hopf et al., 1992). Activation in a similar region has also been reported in a functional imaging study involving the overt matching of facial expressions (George et al., 1993a). Patients with ventral prefrontal lesions, including unilateral lesions, are impaired in face processing of emotional expression independent of the recognition of identity (Hornak et al., 1995). These findings indicate that the ventral prefrontal activation relates to processing of the emotional salience in facial stimuli. However, a more general role in emotional processing remains a possibility as an identical region is activated by the explicit induction of happy or sad moods in normal subjects (Baker et al., 1996). One important region mediating emotional responses which was not activated in the present study was the amygdala. The empirical evidence indicates that fear, derived from visual perception, is mediated via the amygdala (Adolphs et al., 1995). Thus, the role of the amygdala in emotional processing may be quite specific. The use of a single emotional expression, namely happiness, in the present study leaves open the question of whether a separate neural circuitry might be associated with discrete emotions.

Neuropsychological studies indicate that recognition of facial identity and emotion are dissociable processes (Damasio, 1995; Strauss and M oscovitch, 1981; Bowers and Heilman, 1984). Contrasting views are that these processes are mediated by similar or partially dissociable mechanisms (Hansch and Pirozzolo, 1980; DeKosky et al., 1980). Normal subjects and patients with prosopagnosia produce discriminatory skin conductance responses to familiar faces, suggesting covert or implicit recognition (Tranel et al., 1995). In the present study activation of structures additional to areas implicated in overt facial processing suggests these regions may contribute to processing salience in facial stimuli. A previous functional imaging study of faces has described activation within the inferior prefrontal and anterior cingulate cortex in an overt face matching task (Courtney et al., 1996). H owever, the facial stimuli used were not neutral and we suggest that the stimuli of emotional expressions are critical determinants of the magnitude and distribution of the neural response to faces.

Our findings have implications for models of face processing. In prosopagnosia, the mechanism of preserved covert discriminatory responses to previously familiar faces is unknown. One possibility is that an intact ventral and medial prefrontal cortex can maintain the emotional response to familiar faces. At a more general level, the findings providesupport for a theoretical position of separable, though overlapping, neural systems for the processing of facial identity and emotion. The overlapping components involve the right fusiform gyrus while the separable components involve the ventral prefrontal cortex. Finally, the study provides tentative evidence that the neural response to emotional salience in faces is automatic and independent of conscious processing.

\section{REFERENCES}

Adol phs, R., Tranel, D., Damasio, H., and Damasio, A. R. 1995. Fear and the human amygdala. J . Neurosci. 15:5879-5891.

Allison, T., Ginter, H., McCarthy, G., Nobre, A. C., Puce, A., Luby, M., and Spencer, D. D. 1994. Face recognition in human extrastriate cortex. J . Neurophysiol. 71:821-825.

Baker, S., Frith, C. D., and Dolan, R. I. 1996. The interaction of cognition and mood: A PET study. Psychol. Med., in press.

Barrett, S. E., and Rugg, M. D. 1989. Event-related potentials and the semantic matching of faces. Neuropsychol ogia 27:913-922.

Barrett, S. E., Rugg, M. D., and Perrett, D. I. 1988. Event-related potentials and the matching of familiar and unfamiliar faces. Neuropsychologia 26:105-117.

Bauer, R. M. 1986. The cognitive psychology of prosopagnosia. In Aspects of Face Processing (H. Ellis, M. J eeves, and F. Newcombe, Eds.), pp. 253-267. Nijhoff, Dordrecht.

Bench, C. J ., Friston, K. J ., Brown, R. G., Scott, L., Frackowiak, R. S. J ., and Dolan, R. J . 1992. The anatomy of melancholia: Abnormalities of regional cerebral blood flow in major depression. Psychol. Med. 22:607-615.

Bowers, D., and Heilman, K. M. 1984. Dissociation between the processing of affective and nonaffective faces. J . Clin. Neuropsychol. 6:367-379.

Bowers, D., and Heilman, K. M. 1985. Processing of faces by patients with unilateral hemispheric lesions. 1. Dissociation between judge ments of facial affect and facial identity. Brain Cognit. 4:258-272.

Brothers, L. 1990. The social brain: A project for integrating primate behaviour and neurophysiology in a new domain. Concepts Neurosci. 1:27-51.

Bruce, V., and Young, A. W. 1986. Understanding face recognition. Br. J . Psychol . 77:305-327.

Bruyer, R., Laterre, C., Seron, X., Feyereisen, P., Strypstein, E., Pierrard, E., and Rectem, D. 1983. A case of prosopagnosia with some preserved covert remembrance of familiar faces. Brain Cognit. 2:257-284.

Courtney, S. M., Ungerleider, L. G., Keil, K., and Haxby, J . V. 1996. Object and spatial visual working memory activate separate neural systems in human cortex. Cereb. Cortex, in press.

Damasio, A. R. 1995. Descartes Error. Picador, London.

Damasio, A. R., and Van Hoesen, G. W. 1983. Emotional disturbances associated with focal lesions of the frontal lobe. In N europsychology of Human Emotion (K. M. Heilman, and P. Satz, Eds.), pp. 85-110. Guilford Press, New York.

Damasio, A. R., Damasio, H., and Van Hoesen, G. W. 1982. Prosopagnosia: Anatomical basis and behavioural mechanisms. Neurology 32:331-341.

Damasio, A. R., Tranel, D., and Damasio, H. 1990. Individuals with 
sociopathic behavior caused by frontal damage fail to respond autonomically to social stimuli. Behav. Brain Res. 41:81-94.

Damasio, A. R., Tranel, D., and Damasio, H. 1991. Somatic markers and the guidance of behavior: Theory and preliminary testing. In Frontal Lobe Function and Dysfunction ( $\mathrm{H}$. S. Levin, H. M. Eisenberg, and A. L. Benton, Eds.), pp. 217-229. Oxford Univ. Press, New York.

DeHaan, E. H. F., Young, A., and Newcombe, F. 1987. Face recognition without awareness. Cognit. Neuropsychol . 4:385-415.

DeKosky, S. T., Heilman, K. M., Bowers, D., and Valenstein, E. 1980. Recognition and discrimination of emotional faces and pictures. Brain Lang. 9:206-214.

De Renzi, E. 1996. Current issues in prosopagnosia. In Aspects of Face Processing (H. Ellis, M. J eeves, and F. Newcombe, Eds.). Nijhoff, Dordrecht.

Desimone, R., and Gross, C. G. 1979. Visual areas in the temporal lobe of the macaque. Brain Res. 178:363-380.

Devinsky, O., and Luciano, D. 1993. The contributions of the cingulate cortex to human behaviour. In Neurobiology of the Cingulate Cortex and Limbic Thalamus (B. A. Vogt and M. Gabriel, Eds.), pp. 527-556. Birkhauser, Boston.

Ekman, P. 1992. Facial expression of emotion: An old controversy and new findings. Proc. R. Soc. London B 335:63-69.

Ekman, P., and Friesen, W. 1982. Felt, false, and miserable smiles. J . Nonverb. Behav. 6:238-252.

Ekman, P., Friesen, W. V., and Ellsworth, P. 1982. Does the face provide accurate information? In Emotion in the Human Face (P. Ekman, Ed.). Cambridge Univ. Press, Cambridge, UK.

Friston, K. J ., Frith, C. D., Liddle, P. F., and Frackowiak, R. S. J . 1991. Comparing functional (PET) images: The assessment of significant change. J . Cereb. Blood Flow Metab. 11:690-699.

Friston, K. J ., Worsley, K. J ., Frackowiak, R. S. J ., Mazziotta, J . C., and Evans, A. C. 1994. Assessing the significance of focal activations using their spatial extent. Hum. Brain Map. 1:214-220.

Friston, K. J ., Ashburner, J ., Frith, C. D., Poline, J . B., Heather, J . D., and Frackowiak, R. S. J . 1995a. Spatial registration and normalization of images. Hum. Brain Map. 2:165-189.

Friston, K. J ., Holmes, A. P., Worsley, K. J ., Poline, J .-P., Frith, C. D., and Frackowiak, R. S. J . 1995b. Statistical parametric mapping in functional imaging: A general linear approach. Hum. Brain Map. 2:189-210.

George, M. S., Ketter, T. A., Gill, D. S., Haxby, J . V., Ungerleider, L. G., Herscovitch, P., and Post, R. M. 1993a. The role of expression and identity in the face-selective responses in the temporal visual cortex of themonkey. J . Neuropsychiatry Clin. Neurosci. 5:384-394.

George, M. S., Ketter, T. A., Gill, D. S., Haxby, J . V., Ungerleider, L. G., Herscovitch, P., and Post, R. M. 1993b. Brain regions involved in recognising facial emotion or identity: An oxygen-15 PET study. J . Neuropsychiatry Clin. Neurosci. 5:384-393.

Hansch, S., and Pirozzolo, F. J . 1980. Task relevant effects on the assessment of cerebral specialization for facial emotion. Brain Lang. 10:51-59.

Haxby, J ., Horwitz, B., Ungerleider, L. G., Maisog, J . M., Pietrini, P., and Grady, C. L. 1994. The functional organisation of human extrastriate cortex: A PET-rCBF study of selective attention to faces and locations. J . Neurosci. 14:6336-6353.

Hopf, H. C., Muller-Forell, W., and Hopf, N. J . 1992. Localisation of emotional and volitional facial paresis. Neurology 42:1918-1923.

Hornak, J ., Rolls, E. T., and Wade, D. 1996. Face and voice expression identification in patients with emotional and behavioural changes following ventral frontal lobe damage. Neuropsychologia 34:247261.

Kirita, T., and Endo, M. 1995. Happy face advantage in recognizing facial expressions. Acta Psychol. 89:149-163.
Kirouac, G., and Dore, F. Y. 1983. Accuracy and latency of judgment of facial expression of emotions. Percept. Mot. Skills 57:683-686.

Ladavas, E., Cimatti, D., Del Pesce, M., and Tuozzi, G. 1993. E motional evaluation with and without conscious stimulus identification: Evidence from a split-brain patient. Cognit. Emot. 7:95114.

Landis, T., Cummings, J . G., Christen, L., et al. 1986. Are unilateral right posterior lesions sufficient to cause prosopagnosia? Clinical and radiological findings in six additional patients. Cortex 22:243252.

Meadows, J. C. 1974. The anatomical basis of prosopagnosia. J . Neurol. Neurosurg. Psychiatry 37:489-501.

Meltzoff, A. N., and Moore, M. K. 1977. I mitation of facial and manual gestures by human neonates. Science 198:74-78.

Moran, J ., and Desimone, R. 1985. Selective attention gates visual processing in the extrastriate cortex. Science 229:782-784.

Muller-Preuss, P., and J urgens, U. 1976. Projections from the "cingular" vocalization area in the squirrel monkey. Brain Res. 103:2943.

Oldfield, R. C. 1971. The assessment and analysis of handedness: The Edinburgh inventory. Neuropsychologia 9:97-113.

Perrett, D. I., Rolls, E. T., and Caan, W. 1982. Visual neurons responsive to faces in the monkey temporal cortex. Exp. Brain Res. 47:329-342.

Perrett, D. I., Hietanen, J . K., Oram, M. W., and Benson, P. J . 1992. Organization and functions of cells responsive to faces in the temporal cortex. Philos. Trans. R. Soc. London 335:23-30.

Rapsak, S. Z., Comer, J . F., and Rubens, A. B. 1993. Anomia for facial expressions: Neuropsychological mechanisms and anatomical correlates. Brain Lang. 45:233-252.

Rizzo, M., Hurtig, R., and Damasio, A. R. 1987. The role of scanpaths in facial recognition and learning. Ann. Neurol . 22:41-45.

Rolls, E. T., Hornak, J ., Wade, D., and McGrath, J . 1994. Emotionrelated learning in patients with social and emotional changes associated with frontal lobedamage. J . Neurol. Neurosurg. Psychiatry 57:1518-1524.

Sagi, A., and Hoffman, M. L. 1976. Empathetic distress in the newborn. Dev. Psychol. 12:175-176.

Sergent, J ., Ohta, S., MacDonald, B., and Zuck, E. 1994. Segregated processing of facial identity and emotion in the human brain: $A$ PET study. Visual Cognit. 1:349-369.

Shuttleworth, E. C., Syring, V., and Allen, N. 1982. Further observations on the nature of prosopagnosia. Brain Cognit. 1:307-322.

Strauss, S., and Moscovitch, M. 1981. Perception of facial expressions. Brain Lang. 13:308-332.

Tranel, D., and Damasio, A. R. 1985. Knowledge without awareness. Science 228:1453-1454.

Tranel, D., Damasio, H., and Damasio, A. R. 1995. Double dissociation between overt and covert face recognition. J . Cognit. Neurosci. 7:425-432.

Uhl, F., Lang, W., Speith, F., and Deecke, L. 1990. Negative cortical potentials when classifying familiar and unfamiliar faces. Cortex 26:157-161.

Von Cramon, D., and J urgens, U. 1983. The anterior cingulate cortex and the phonatory control in monkey and man. Neurosci. Biobehav. Rev. 7:423-425.

Wilson, F. A. W., Scalaidhe, S. P., and Goldman-Rakic, P. S. 1993. Dissociation of object and spatial processing domains in primate prefrontal cortex. Science 260:1955-1958.

Young, A. W., Newcombe, F., de Haan, E. H. F., Small, M., and Hay, D. C. 1993. Face perception after brain injury: Selective impairments affecting identity and expression. Brain 116:941-959. 